\title{
AN EFFICIENT NUMERIC INDEXING TECHNIQUE FOR MUSIC RETRIEVAL SYSTEM
}

\author{
Chuan-Wang Chang and Hewijin Christine Jiau \\ Department of Electrical Engineering \\ National Cheng Kung University, Tainan City 701, Taiwan \\ E-mail: chuan@ee.ncku.edu.tw and jiauhjc@mail.ncku.edu.tw
}

\begin{abstract}
Space requirement for storing indexes and performance for query processing are two critical issues in music information retrieval (MIR) system. To overcome difficulties in variable length of queries and enhance efficiency of music retrieval, we propose an effective and efficient numeric indexing structure. It differs greatly from preexisting researches in textual indexing techniques. We show how the development of this framework has been motivated and demonstrate how the technique may be naturally applied to solve this two fundamental MIR issues. Experiments are performed to compare our method with previous solutions. The results show that our method is more scalable and economical than previous methods. The method we proposed can achieve dramatically and significantly improvement in saving time and storing space for retrieving and indexing.
\end{abstract}

\section{INTRODUCTION}

The rapid growth of computer science has resulted in a rapid increase of the size of digital multimedia data collections and greatly increases the availability of multimedia contents to user. Digital music is one of the most popular data types distributed by the Internet. Large web-based music collections are continuing to grow in size exponentially. Clearly, the ability to effectively and efficiently manage the great amount of music data is desirable. Music information retrieval (MIR) system is expected to be such a system that has powerful ability to handle music data and to satisfy the requirements of users.

Ghias, et al. [1] transform a music object into a string which consists of three kinds of symbols ("U", "D" and "S" stand for a note which is higher than, lower than, or equal to the previous note, respectively). The string can be regarded as a coarse melodic contour of the music. Then, the problem of music retrieval is transformed into string matching.

No matter how the pre-existing methods [1-4] work, they view the music object as a string, and the processes of indexing and retrieval are by means of text-related techniques.

Suffix tree $[5,6]$ is a tree-based index structure which is widespread used in MIR systems. If $T=t_{1} t_{2} t_{3} \ldots t_{i} \ldots t_{n}$ is a string, then $T_{i}=t_{i} t_{i+1} \ldots t_{n}$ is the suffix of $T$ that starts at position $i$. It means a suffix is a substring that includes the final character of the string. Figure 1 shows a suffix tree for the string $T=$ 'abababc'. We can find that 'c', 'bc', 'abc', 'babc', 'ababc', 'bababc', and 'abababc' are suffixes of $T$. The leaf nodes indicate the starting position of the corresponding suffix.

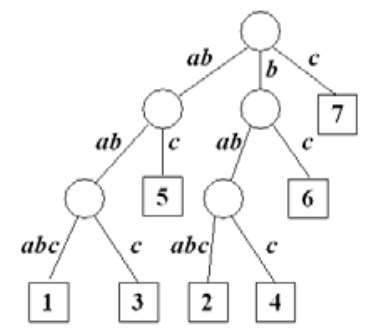

Figure 1. The suffix tree representation of $T=$ 'abababc'.

$N$-gram is another technique widely used in MIR systems [7, 8, 9]. The sequence of characters (symbols) is divided into overlapping constant-length subsequences (substrings). A string formed from $n$ adjacent characters within text is called an n-gram. For example, the string 'abababc' comprises the following 4-grams: 'abab', 'baba', 'abab' and 'baba'. Downie [7] adopted this technique to evaluate his MIR system. The songs were converted to a sequence of intervals and then using a gliding window to fragment the sequence into overlapping length- $n$ substrings. These $n$-grams were then encoded as text words. Hence, each song can be viewed as a text document, and a preexisting text search engine can be used for text retrieval.

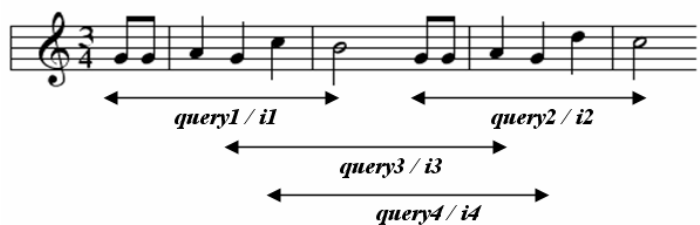

Figure 2. An excerpt of 'Happy Birthday to You'.

However, it is quit obvious that the memory usage for storing indexes by using suffix tree or $n$-gram method is very large. The indexes are full of redundancies. Since most constituents of suffix tree and $n$-grams are unnatural and dissimilar to human habit. People tend to submit a perceptually meaningful query segment (phrase). For example, Figure 2 shows an excerpt of a familiar song-'Happy Birthday to You'. The queryl and query2 are two 
possible meaningful queries. Because they seem to be two clear phrases, while query 3 and query 4 are unlikely to be hummed. Unfortunately, using $n$-gram method (6-gram in this case), i3 that is composed of 'sol-do-si-sol-sol-la' and $i 4$ that is composed of 'do-si-sol-sol-la-sol' are included in indexes. This phenomenon indicates that the indexes contain many unnecessary data. The space for storing indexes will become smaller when redundancies pruning is conducted.

To reduce memory space requirement and enhance query processing, in this paper, we propose an efficient indexing approach based on the advantages of numeric indexing technique. Instead of textual indexing techniques, the performance of memory usage and retrieval for MIR by using numeric indexing techniques is better.

The rest of this paper is organized as follows. In Section 2, we introduce terminologies and notations used in this paper. Section 3 describes the basic concepts of our numeric indexing strategies. In Section 4, we demonstrate the accomplished experimental results. Finally, in Section 5, we summarize this paper.

\section{TERMINOLOGY AND NOTATION}

To illustrate the proposed indexing strategies, introduce terminologies and notations used in this paper is necessary. In our method, music are segmented to phrases [10] and transformed into ternary melodic contours. Figure 3 sketches several terminologies. The definitions of specific terms used in this paper are as follows.

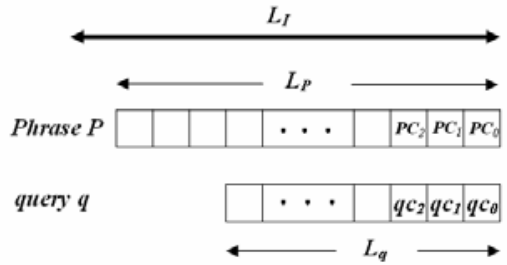

Figure 3. A simple diagram shows several terminologies.

- $P$ : melodic contour of a music phrase that intends to be indexed

- $P C_{i}$ : the $i$ th digit of phrase contour $P$

- $q$ : a query submitted by user

- $q c_{i}$ : the $i$ th digit of query contour $q$

- $L_{I}$ : the maximum index length of MIR system

- $L_{P}$ : the length of phrase $P$

- $L_{q}$ : the length of query $q$

A music phrase is denoted as a 5-tuple (I $I_{P R E}, I_{S U F}$, length, Song_id, Phrase_num), where

- $I_{P R E}$ indicates the first index of music phrase $P$.

- $I_{S U F}$ indicates the second index of music phrase $P$

- length denotes the length of music phrase $P$.

- Song_id represents the identification number of the music.

- Phrase_num is the serial number of segmented phrase that belongs to the music with the number of Song_id.

For example, suppose a phrase $P$ with length of 4 is the first phrase that extracted from the song $w 2 s 1$, the first index is 3 and the second index is 4 , then the phrase $P$ is denoted as $(3,4, w 2 s 1, p 1)$.

\section{PROPOSED NUMERIC INDEXING TECHNIQUE}

In order to decrease the size of indexes and accelerate music retrieval, we introduce the advantage of numeric indexing technique and propose an effective and efficient indexing structure. The essence of the proposed numeric indexing scheme is adoption of perceptually meaningful phrase to establish index unlike suffix tree and $n$-gram methods. Besides, the proposed method is regardless of phrase length, that is, it needs not to divide the phrases into overlapping constant-length subsequences in advance.

Figure 4 shows the basic idea of the proposed numeric indexing structure. The extracted representative fragments (music phrases) are indexed by $f(c)$, where $f()$ is a mapping function while $c$ is the melodic contour of music phrase. That is, a phrase with key $c$ maps to slot $f(c)$. Here, phrases have the same $f(c)$ are stored in the same slot. The submitted query is used to retrieve phrases that have the same $f(c)$.

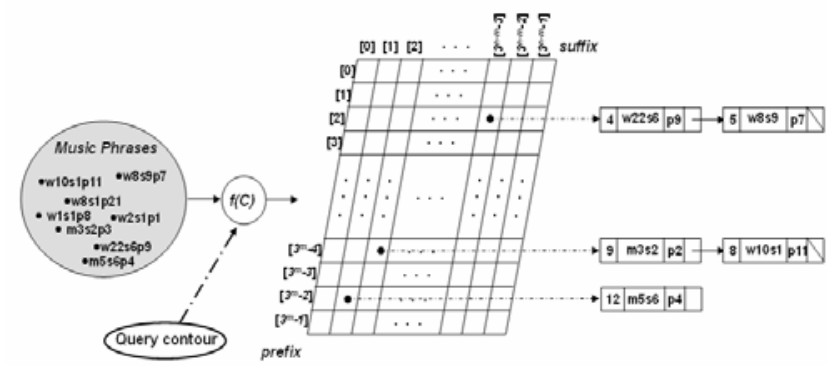

Figure 4. Basic idea of the proposed indexing structure.

Because of the different segmentation for phrases between user's cognition and MIR system's judgment, user may submit a self-determined phrase that differs from original phrase segmented by MIR system.

To handle various kinds of query cases, we adopt dual index structure. The first index $I_{P R E}$ and the second index $I_{S U F}$ are derived from the phrase $P$ directly.

In general, we can constrain the total length $L_{I} \leq L_{I_{P R E}}+L_{I_{S U F}}$ of index to prevent the explosively increase of space requirement.

The indexing functions are defined as

$$
\begin{aligned}
& I_{P R E}=\boldsymbol{f}\left(\boldsymbol{P}_{P R E}\right)=\sum_{i=0}^{i=L_{I_{P R E}}-1} P C_{L_{P}-1-i} \times 3^{L_{I_{P R E}}-1-i} \\
& \boldsymbol{I}_{\text {SUF }}=\boldsymbol{f}\left(\boldsymbol{P}_{\text {SUF }}\right)=\sum_{i=0}^{i=L_{I S U F}-1} \boldsymbol{P} \boldsymbol{C}_{\boldsymbol{i}} \times 3^{\boldsymbol{L}_{I_{\text {SUF }}}-1-\boldsymbol{i}}
\end{aligned}
$$

To show feasibility and advantages of the proposed methods, all kinds of cases are considered and comprehensive discussions are made in the following.

Case 1. If $q=P, L_{q} \leq L_{I_{P R E}}$ and $L_{q} \leq L_{I_{S U F}}$

Figure 5 shows the concept of this case. According to Eq.(1) and Eq.(2), phrase $P$ is indexed as

$$
\begin{aligned}
& I_{P R E}=\boldsymbol{f}\left(\boldsymbol{P}_{P R E}\right)=\sum_{i=0}^{i=L_{P}-1} P C_{L_{P}-1-i} \times 3^{L_{I P R E}-1-i} \\
& \boldsymbol{I}_{\text {SUF }}=\boldsymbol{f}\left(\boldsymbol{P}_{\text {SUF }}\right)=\sum_{i=0}^{i=L_{P}-1} \boldsymbol{P} \boldsymbol{C}_{\boldsymbol{i}} \times 3^{\boldsymbol{L}_{\text {ISUF }}-1-\boldsymbol{i}}
\end{aligned}
$$


Since $q=P$, we have

$$
f\left(q_{P R E}\right)=f\left(P_{P R E}\right) \text { and } f\left(q_{S U F}\right)=f\left(P_{S U F}\right) .
$$

Thus, phrase $P$ can be exactly retrieved.

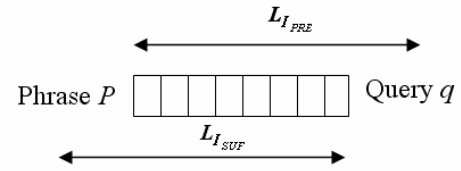

Figure 5. A diagram to explain Case 1.

Case 2. If $q=P$ and $\boldsymbol{L}_{q} \leq\left(\boldsymbol{L}_{L_{P R E}}+\boldsymbol{L}_{\boldsymbol{L}_{S U F}}-\boldsymbol{L}_{\left(\boldsymbol{L}_{P R E} \cap \boldsymbol{L}_{S U F}\right)}\right)$

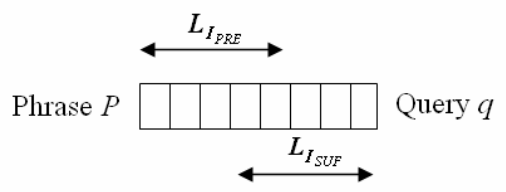

Figure 6. A diagram to explain Case 2.

In this case, phrase $P$ is indexed by Eq.(1) and Eq.(2). Since $q=P$, we have

$$
f\left(q_{P R E}\right)=f\left(P_{P R E}\right) \text { and } f\left(q_{S U F}\right)=f\left(P_{S U F}\right) .
$$

Thus, phrase $\mathrm{P}$ can also be exactly retrieved.

Case 3. if $\boldsymbol{L}_{\boldsymbol{P}}>\boldsymbol{L}_{\boldsymbol{I}}=\left(\boldsymbol{L}_{\boldsymbol{I}_{P R E}}+\boldsymbol{L}_{\boldsymbol{I}_{S U F}}\right)$ and $q=P$

Consider Figure 7, it illustrates the case when the length of original phrase is longer than the length of systemallowed index. Due to the limitation of index length, phrase $P$ is indexed as

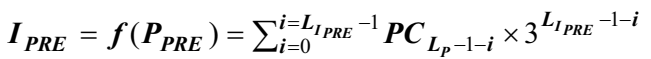

$$
\begin{aligned}
& \boldsymbol{I}_{\text {SUF }}=\boldsymbol{f}\left(\boldsymbol{P}_{\text {SUF }}\right)=\sum_{i=0}^{i=L_{\text {ISUF }}-1} \boldsymbol{P} \boldsymbol{C}_{\boldsymbol{i}} \times 3^{\boldsymbol{L}_{\text {ISUF }}-1-\boldsymbol{i}} \\
& \text { If } q=P \text {, we have } \\
& \boldsymbol{f}\left(\boldsymbol{q}_{P R E}\right)=\sum_{i=0}^{i=L_{I_{P R E}}-1} \boldsymbol{q} c_{L q-1-i} \times 3^{L_{I P R E}-1-i}=\boldsymbol{f}\left(P_{P R E}\right) \\
& \boldsymbol{f}\left(\boldsymbol{q}_{\text {SUF }}\right)=\sum_{i=0}^{i=L_{\text {ISUF }}-1} \boldsymbol{q} \boldsymbol{c}_{\boldsymbol{i}} \times 3^{\boldsymbol{L}_{\text {ISUF }}-1-\boldsymbol{i}}=\boldsymbol{f}\left(\boldsymbol{P}_{\text {SUF }}\right) .
\end{aligned}
$$

Hence, phrase $P$ can be exactly retrieved.

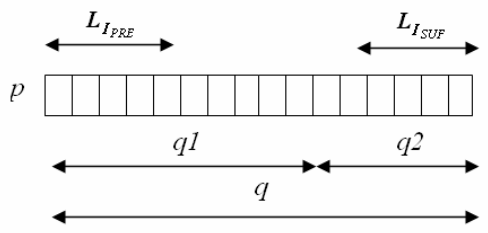

Figure 7. A diagram to explain Case 3.

Case 4. if $\boldsymbol{L}_{\boldsymbol{P}}>\boldsymbol{L}_{\boldsymbol{I}}=\left(\boldsymbol{L}_{I_{P R E}}+\boldsymbol{L}_{I_{S U F}}\right)$ and $\boldsymbol{L}_{q 1}<\boldsymbol{L}_{P}$

This case is similar to Case 3 , but the query is $q 1$. Since $\boldsymbol{f}\left(\boldsymbol{q} 1_{P R E}\right)=\sum_{i=0}^{i=L_{I_{P R E}}-1} q c_{L_{q 1}-1-i} \times 3^{L_{I_{P R E}}-1-i}=f\left(P_{P R E}\right)$, the MIR system will return many recalls that have the same prefix as $q 1$, of course including phrase $P$, in spite of $\boldsymbol{f}\left(\boldsymbol{q} 1_{\text {SUF }}\right) \neq \boldsymbol{f}\left(\boldsymbol{P}_{\text {SUF }}\right)$. Figure 8 shows the concept of this case. The shade indicates the possible retrieved phrases.

Case 5. if $\boldsymbol{L}_{P}>\boldsymbol{L}_{I}=\left(\boldsymbol{L}_{I_{P R E}}+\boldsymbol{L}_{I_{S U F}}\right)$ and $\boldsymbol{L}_{q 2}<\boldsymbol{L}_{P}$
This case is similar to Case 4 , if we assume the submitted query is $q 2$, then from Eq.(1) we get:

$$
\boldsymbol{f}\left(\boldsymbol{q} 2_{\mathrm{PRE}}\right)=\sum_{i=0}^{i=L_{I_{P R E}}-1} \boldsymbol{q} \boldsymbol{c}_{L_{P}-1-i} \times 3^{L_{I_{P R E}}-1-i} \neq \boldsymbol{f}\left(\boldsymbol{P}_{P R E}\right)
$$

and from Eq.(2) we obtain:

$$
f\left(q 2_{\text {SUF }}\right)=\sum_{i=0}^{i=L_{\text {SUF }}-1} q c_{i} \times 3^{L_{\text {ISUF }}-1-i}=f\left(P_{\text {SUF }}\right) .
$$

In this case, system will return many recalls that have the same suffix as $q 2$, of course including phrase $P$, in spite of $f\left(q 1_{\text {PRE }}\right) \neq f\left(P_{\text {PRE }}\right)$. Figure 9 shows the concept of this case.

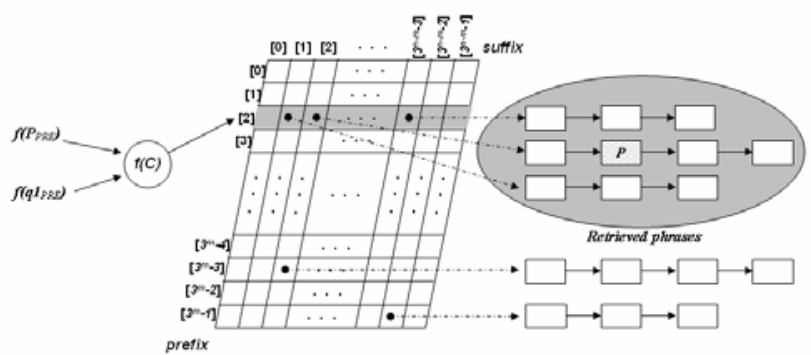

Figure 8. The concept of Case 4.

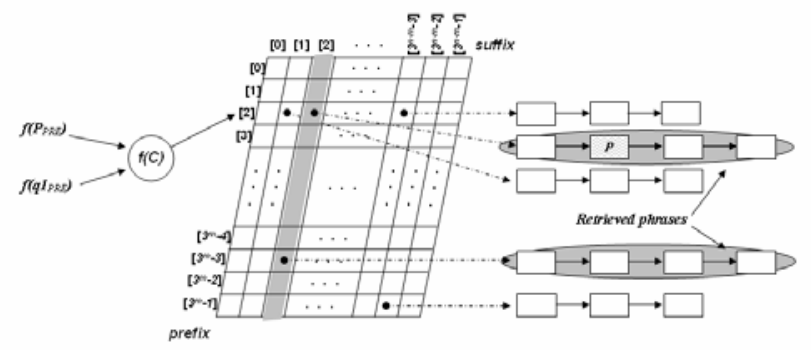

Figure 9. The concept of Case 5.

\section{EXPERIMENTAL RESULTS}

To evaluate the effectiveness and efficiency of the proposed indexing method, we performed an extensive performance study of three methods: n-gram, suffix tree, and the proposed numeric indexing, with various kinds of index length.

We collected more than 1,000 MIDI files from the public domain to form the music database. To construct the music database, the melody data have to be extracted first. After melody extraction, the music database consists of over 374,000 notes. We transformed each song into note string. To evaluate the proposed numeric indexing approach, we segmented the melodic contours of song into perceptually meaningful phrases [10]. There are about 18,000 phrase stored in music database.

For $n$-gram method, we transform note strings into melodic contours. Consider a melodic contour with length of $n$. We partition the melodic contours to $n-m+1$ successive segments with specific length $m$ and construct interval blocks for indexing [9]. Then, information of these melodic segments, such as the title and the occurrence position of the song, are stored in interval blocks. In exactly matching, we retrieve songs that contain identical piece with 
the query. In similarity matching, we retrieve songs that have the same melodic contour of query.

Table 1: Performance Comparison of Different Methods

\begin{tabular}{|c|c|c|c|c|}
\hline Per & formance & \begin{tabular}{|c|} 
Memory \\
Consumption \\
of Index \\
Construction \\
(KB)
\end{tabular} & $\begin{array}{l}\text { Elapsed } \\
\text { Time for } \\
\text { Exactly } \\
\text { Matching } \\
\text { (Sec.) }\end{array}$ & $\begin{array}{l}\text { Elapsed } \\
\text { Time for } \\
\text { similarity } \\
\text { Matching } \\
\text { (Sec.) }\end{array}$ \\
\hline & 4-gram & 1,505 & 6.6226 & 9.0486 \\
\hline & 5-gram & 1,683 & 4.9723 & 6.7692 \\
\hline & 6-gram & 1,894 & 2.9015 & 3.6532 \\
\hline n-gram & 7-gram & 2,083 & 1.7098 & 2.1828 \\
\hline & 8-gram & 2,229 & 0.9772 & 1.2859 \\
\hline & 9-gram & 2,338 & 0.4537 & 0.7350 \\
\hline & 10-gram & 2,419 & 0.2324 & 0.4566 \\
\hline Suffix & Tree & 78,160 & 1.8642 & 3.4325 \\
\hline & 43 & 242 & $<<0.001$ & 0.063 \\
\hline & $4 \quad 4$ & 260 & $<0.001$ & 0.031 \\
\hline & $4 \_5$ & 286 & $<0.001$ & 0.016 \\
\hline & $4 \_6$ & 333 & $<0.001$ & 0.015 \\
\hline & $4 \_7$ & 452 & $<0.001$ & 0.001 \\
\hline Our Method & 544 & 289 & $<0.001$ & 0.015 \\
\hline$\left(I_{P R E \_} I_{S U F}\right)$ & $5 \quad 5$ & 336 & $<0.001$ & 0.016 \\
\hline & $5 \_7$ & 802 & $<0.001$ & 0.016 \\
\hline & 644 & 342 & $<0.001$ & 0.016 \\
\hline & $6 \_6$ & 802 & $<0.001$ & 0.001 \\
\hline & 74 & 447 & $<0.001$ & 0.001 \\
\hline & 75 & 730 & $<0.001$ & 0.016 \\
\hline
\end{tabular}

For suffix tree method, the goal of exactly matching is to traverse from the root of suffix tree to a specific leaf node according to the query string. Under the limitation of the depth for searching, the similarity matching is to return all leaves under a specific internal node.

For the proposed numeric indexing approach, the task of exactly matching is to retrieve all phrases that have both the same prefix and suffix as query. In addition, the task of similarity matching is to retrieve all phrases that have either the same prefix or suffix as query.

All experiments were conducted on a $2.8 \mathrm{GHz}$ Intel Pentium 4 PC with $1 \mathrm{G}$ main memory, running Microsoft Windows XP professional. Table 1 demonstrates the memory usage for indexing and elapsed time for retrieving. In the aspect of memory usage, the suffix tree method is the worst (76 MB) because it enumerates all suffixes. The disk space cost by the $n$-gram method in various lengths are about 1 2 MB. The proposed numeric indexing method can dramatically reduce the space requirement for storing indexes. In the aspect of retrieving performance, we randomly select 20 music excerpts as queries for further validation. Compare to these methods, using the proposed numeric indexing method can greatly decrease the elapsed time for exactly matching and similarity matching.

The depth of indexing is the most important factor which dominates the performance of that using suffix tree method. In the proposed numeric indexing approach, no matter what the index length is, the elapsed time for exactly matching is quite small $(<0.001$ second).

\section{CONCLUSIONS}

In this paper, we mainly focus on two issues: the size of indexes and the time for retrieving music. Because the indexes are full of redundancies by using pre-existing indexing methods, we adopt music phrase as the basic unit for further processing. In addition to overcome the difficulties caused by the difference between phrase extracted by system and phrase segmented by user, we introduced numeric indexing approach. Besides, the proposed indexing approach not limit to the length of phrase for indexing or querying. The experimental results demonstrate that our method performs more efficient and effective compared with the pre-existing methods.

\section{REFERENCES}

[1]A. Ghias, H. Logan, D. Chamberlin, and B.C. Smith, "Query by Humming : Musical Information Retrieval in an Audio Databases," Proc. of the 3rd ACM International Conference on Multimedia, pp. 231-236, 1995.

[2]A L.P. Chen, M. Chang, J. Chen, J.L. Hsu, C.H. Hsu, and S.Y.S. Hua, "Query by Music Segments: An Efficient Approach for Song Retrieval, ” Proc. of IEEE International Conference on Multimedia and Expo, pp. 873-876, 2000.

[3]J.L. Hsu, C.C. Liu, and A.L.P. Chen, "Discovering Nontrivial Repeating Patterns in Music Data," IEEE Transactions on Multimedia, pp. 311-325, 2001.

[4]W. Lee, and A.L.P. Chen, "Efficient Multi-Feature Index Structures for Music Data Retrieval," Proc. of SPIE Conference on Storage and Retrieval for Image and Video Databases, pp. 177-188, 2000.

[5]E. M. McCreight, "A Space-Economical Suffix Tree Construction Algorithm," Journal of Algorithms, vol. 23(2), pp. 262-272, 1976.

[6]E. Ukkonen, "On-line Construction of Suffix Trees," Algorithmica, Vol. 14(3) pp. 249-260, 1995.

[7]S. Downie and M. Nelson, "Evaluation of a simple and effective music information retrieval method," Proc. of 23rd Annual International ACM SIGIR Conference on Research and Development in Information Retrieval, pp. 73-80, 2000.

[8]C. L. Yip and B. Kao, "A Study on N-gram Indexing of Music Features," Proc. of IEEE International Conference on Multimedia and Expo, pp. 869-872, 2000.

[9]C.W. Chang, C.Y. Chang and H.C. Christine Jiau, "A Practical Music Retrieval System Based on Sliding Melodic Contour," Proc. of International Computer Symposium, pp. 1162-1167, 2004.

[10]C.W. Chang and H.C. Christine Jiau, "Representative Music Fragments Extraction by Using Segmentation Techniques," Proc. of International Computer Symposium, pp. 1156-1161, 2004. 